\title{
El destino del Bono de Desarrollo Humano y el efecto en la calidad de vida de los grupos vulnerables de la Provincia de Cotopaxi
}

\section{The destiny of the Human Development Bond and its effect in the quality of life of vulnerable groups in the Province of Cotopaxi}

\author{
MSc, Diego Mauricio Bonilla Jurado ${ }^{1}$ \\ administracion@bhconsultores.com \\ MSc, Claudio Homero Tutín Navas ${ }^{2}$ \\ claudio7tutin@hotmail.com \\ MSc, Henry Robert Lopez Nuñez ${ }^{3}$ \\ henryrobertlopez@gmail.com
}

Recibido: 1/07/2019; Aceptado: 1/09/2019

\begin{abstract}
RESUMEN
El objetivo del presente trabajo es analizar desde una perspectiva cualitativa la mejora de la calidad de vida por medio del bono de Desarrollo Humano, implementado por el gobierno para las personas que se hallan dentro del índice de vulnerabilidad y pobreza mencionado anteriormente o encontrarse dentro de los criterios de protección. Para ello se utilizó un alcance descriptivo correlacional dado que se detalló los factores que inciden en la calidad de vida y el destino del monto que se utilizaba. La población está estructurada por madres solteras, personas con capacidades especiales y adultos mayores en el año 2017 en la provincia de Cotopaxi con un total de 43.000 beneficiarios. Bajo este contexto y como método para verificar la hipótesis planteada se utilizó el Ji cuadrado, donde se obtuvo un X2 calculado de 45,6 y un X2 tabulado de 18,30 . Concluyendo que el bono de desarrollo humano no mejora la calidad de vida de las personas involucradas en el estudio.
\end{abstract}

Palabras Clave: bono de desarrollo humano, calidad de vida, personas vulnerables, pobreza

\footnotetext{
${ }^{1}$ Bh Consultores, Ambato, Ecuador

2 Universidad Técnica de Ambato, Ecuador

3 Tecnológico Superior Bolívar, Quito, Ecuador
}

Revista científica Ciencia y Tecnología Vol 19 No 24 págs. 9 - 19 http://cienciaytecnologia.uteg.edu.ec 


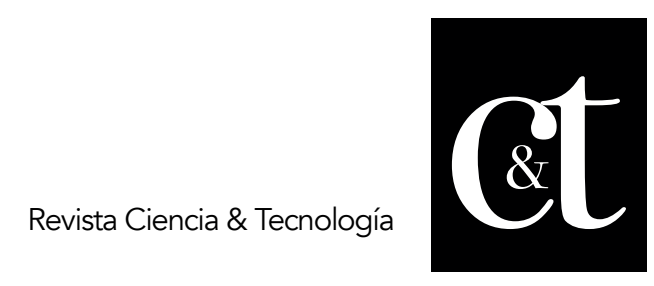

No. 24, 31 de octubre de 2019

ISSN impreso: 1390 - 6321

ISSN online: 2661 - 6734

\begin{abstract}
The objective of this paper is to analyze, from a qualitative perspective, the improvement in the quality of life through the Human Development Bond implemented by the government for people who are within the vulnerability and poverty index, or within the criteria of protection. For this, a correlational descriptive scope is used, since it has been detailed the factors that affect the quality of life and the destination of the amount that was used. The population was structured by single mothers, people with special abilities and older adults in the year 2017 in the province of Cotopaxi, with a total of 43,000 beneficiaries. Under this context and as a method to verify the hypothesis, the square Chi was used, where an X2 of 45.6 and a tabulated X2 of 18.30 was obtained. Concluding that the human development bonus does not improve the quality of life of the people involved in the study.
\end{abstract}

Keywords: human development bonus quality of life, vulnerable people, poverty

\title{
Introducción
}

Lo que todo hombre quiere es tener y mejorar su calidad de vida independientemente del esfuerzo que están dispuesto a hacerlo, a lo largo de los años es indispensable discutir la calidad de vida sin hacer referencia al hombre y descuidar las aportaciones de la Antropología, la Psicología y la Filosofía, que definen a su vez la naturaleza del hombre, su actividad intelectual, la existencia del alma sustancial individual y la relación con los principios y valores que se fomenta en la sociedad. Sin embargo, los gobiernos de los países fomentan campañas para tratar de mejorar la calidad de vida de las personas especialmente de aquellas que se encuentran en los extractos sociales más bajos de la sociedad y que necesitan algún tipo de ayuda para su subsistencia.

Por otro lado, el gobierno central a través de los programas sociales busca el incremento de los ingresos de las familias de bajos recursos para que logren satisfacer sus necesidades básicas, incluye un componente que busca fomentar y promover el desarrollo del capital humano y mejorar su calidad de vida y la de sus familiares con lo que se lograría interrumpir el ciclo intergeneracional de la pobreza.

El bono de desarrollo humano $(\mathrm{BDH})$ consistió en un programa de transferencias monetarias condicionadas que buscaba combatir la pobreza de manera estructural y coyuntural. Por un lado, de forma estructural, establece la obligatoriedad en la asistencia de los hijos de los beneficiarios a los centros educativos y de salud permitiendo mejorar sus condiciones de vida, aumentar su capital humano e interrumpir la transmisión intergeneracional de la pobreza, mientras que, por el otro lado, coyunturalmente, entrega un monto económico para mantener los niveles de consumo mínimo de las familias (Roura Camacho, 2016). 


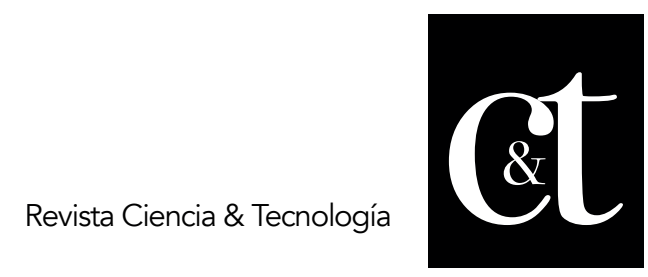

No. 24, 31 de octubre de 2019

ISSN impreso: 1390 - 6321

ISSN online: 2661 - 6734

\section{Desarrollo}

Marco Teórico

La calidad de vida se debe mirar desde dos puntos: la parte individual (privada) y la parte colectiva (pública); desde lo individual o privado es importante mirar: percepción, sentido de vida, utilidad, valoración, felicidad, satisfacción de necesidades y demás aspectos subjetivos que son difícilmente cuantificables, pero que hacen que una vida tenga calidad con responsabilidad moral. (Cardona \& Agudelo, 2005). La calidad de vida es un concepto de creciente interés tanto en la sociedad como en el mundo académico. Su ambigüedad conceptual ha dado lugar a numerosos debates relacionados con su definición y con la metodología para estudiarla. Los trabajos empíricos intentan superar las discusiones con la finalidad de analizar las condiciones de vida de la población para que los agentes con capacidad de decisión adopten las medidas necesarias para su mejora. (Celemin, Mikkelsen, \& Velázquez, 2015) La calidad de vida también depende de los que tienen ingresos más elevados, tiene a existir una satisfacción mayor con la forma de vivir, en la medida que la pareja es la forma más habitual de convivencia, y también se encuentran más satisfechos con las relaciones sociales y con el entorno, contraponiéndose con aquellos mayores que pueden tener una situación más marginal, económica y socialmente, quienes no encuentran tanta satisfacción en la vida que desarrollan (Rodriguez, Rojo, \& Fernández, 2011).

El desarrollo, en gran medida, ha dejado de relacionarse estrictamente con el crecimiento económico de los países, para ampliar su espectro incluyendo elementos que son igualmente importantes, como las capacidades humanas, el entorno, la cultura e incluso el medio ambiente, lo que ha permitido que se hable de desarrollo humano y de desarrollo humano sostenible, las posturas de pensamiento actuales abogan por una visión multidimensional del mismo que abarque lo humano, lo económico, lo ecológico y lo social (Arias Cortés, 2013).

El papel del sector público cobra especial relevancia a la hora de facilitar la creación, y no la imposición, de otro modo de economía. Es importante destacar que esta propuesta no defiende más sector público, sino "otro sector público". Una administración pública que ceda protagonismo a la sociedad civil, mediante una mayor participación democrática y la facilitación de espacios para el desarrollo de proyectos colectivos. Esta administración pública cuenta además con otras herramientas básicas como son la normativa, el gasto público y la fiscalidad para crear incentivos que favorezcan el fomento del bien común con la participación democrática, construcción participativa del índice, modificación de la cultura de la gestión pública, concienciación ciudadana, creación de redes empresariales y sociales fundamentadas en la cooperación y no en la competencia, etc. (Gómez Álvarez, Morales Sánchez, \& Rodriguez Morilla, 2017).

El incremento en el número de emprendimientos es una muestra del cambio en la actitud hacia actividades comerciales que generan un mayor valor agregado y el empoderamiento que esto genera. Así mismo, esto implica el abandono paulatino de actividades primarias orientadas principalmente al autoconsumo, por lo tanto debe existir acceso a nuevos mercados fortaleciendo e incrementando el mercado local por medio de la puesta en marcha de políticas vinculadas con la compra a proveedores 


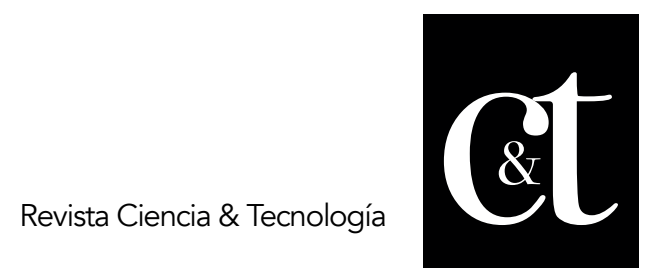

No. 24, 31 de octubre de 2019

ISSN impreso: 1390 - 6321

ISSN online: 2661 - 6734

locales y mecanismos de financiamiento, tanto para colaboradores como emprendedores de la región. La creación de empresas que representen un mecanismo alternativo a los programas de desarrollo rural tradicionales, que encabezan entidades gubernamentales y organismos internacionales que no han producido los resultados esperados (Vázquez Maguirre, 2014).

Es importante reconocer que las políticas económicas neoliberales nos han conducido a un sistema donde las desigualdades sociales han aumentado. Estas desigualdades implican pobreza, desempleo y enfermedad, entre otras desventajas que menguan la calidad de vida y la sustentabilidad de los sistemas en que subsistimos. La modernización no ha sido un proceso integral y practicable, sino truncado, fragmentario y perturbador, con muy serios desequilibrios que han ensombrecido el panorama y cuyos síntomas se han manifestado en una desarticulación social de la eufemísticamente llamada "modernidad" que ha hecho desaparecer el sentido de lo comunitario y se ha evidenciado en las dificultades para lograr una afinidad social, y también ha conllevado un cambio fundamental en la forma de convivencia (Cantú Martínez, 2015).

Bono de Desarrollo

El Bono de Desarrollo Humano es el programa pilar del sistema de protección social del Ecuador. Creado con el nombre de Bono Solidario en el mes de septiembre de 1998, fue diseñado como una medida de compensación social otorgada por el Estado ante la eventual reducción y eliminación de los subsidios generalizados energéticos altamente regresivos en la gasolina, el gas y la electricidad. En su espíritu inicial, se constituyó como un subsidio directo que tenía el objeto de proteger el consumo mínimo de las personas pobres del país que se verían afectadas por esta eliminación de subsidios. Posteriormente, y dada la crisis económica-política que afectó al país entre 1999-2000, se constituyó como la principal herramienta del gasto social del país para combatir los efectos de la crisis y proteger a la población más vulnerable. (Martínez Dobronsky \& Rosero Moncayo, 2015) Tomando en cuenta que el BDH, constituye el programa de transferencias monetarias directas más grande de América Latina en lo que se refiere al porcentaje de población al que beneficia el $40 \%$ del total de hogares y que fueron condicionados para educación y salud, y que cada uno de ellos implica diferentes objetivos de política y de evaluación de impacto (Martínez Dobronsky \& Rosero Moncayo, 2015).

Los caminos hacia el desarrollo sostenible

La tarea fundamental para el tránsito al desarrollo sostenible está dada por el mantenimiento o aumento del capital natural del que dependen las economías. Para ello se requiere de inversiones en capital. Un mejoramiento en los instrumentos de política podría promover la inversión en capital natural. Para el efecto, se debería apelar a reformas regulatorias que apoyen el uso apropiado de los incentivos financieros, legales y sociales. También habría que incluir impuestos a la contaminación y a la explotación del capital natural, así como la posibilidad de conceder permisos para emisiones de gases que sean intercambiables en un mercado entre productores industriales (Troya, 2014).

Las propuestas de la economía para propender al desarrollo sostenible son 


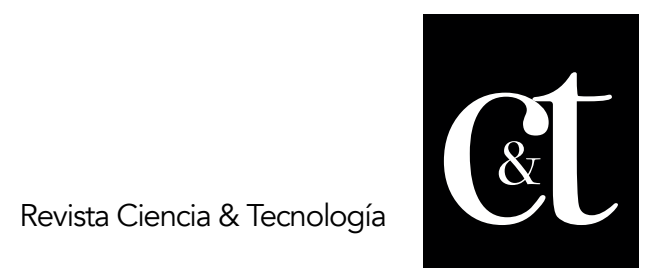

No. 24, 31 de octubre de 2019

ISSN impreso: 1390 - 6321

ISSN online: 2661 - 6734

coincidentes en tanto en cuanto reconocen en los mecanismos de mercado una herramienta que permite una asignación más eficaz de los recursos, lo cual contribuye a la eficiencia de los procesos económicos y, por tanto, disminuye el impacto de éstos. Ambas concepciones también admiten las imperfecciones existentes en los mercados como para proponer correctivos gubernamentales a las distorsiones que se producen en la economía.

Asimismo, ninguna de las dos concepciones aboga por una economía estancada, es decir sin ningún crecimiento. Sin embargo, mientras la primera estima viable un crecimiento en el tiempo, la segunda alerta sobre la imposibilidad de sostener el crecimiento en el largo plazo y aboga más por una sostenibilidad del desarrollo en la cual es posible llegar a un mejoramiento en la calidad de vida sin que necesariamente se produzca un incremento en la cantidad de recursos consumidos (Troya Rodríguez, 2014).

\section{Metodología}

La investigación tiene enfoque cuantitativo ya que se obtendrán datos cuantitativos debido a que se toman datos estadísticos como el número de beneficiarios, edades, etc. y permite conocer la perspectiva estructural y verificar el estilo de vida de los más vulnerables en los diferentes sectores. Se utilizó un alcance descriptivo donde se identificó los factores que inciden en la calidad de vida de los grupos que son beneficiarios del Bono de Desarrollo Humano en el sector rural de la Provincia de Cotopaxi. Como población y muestra para la investigación se tomó datos de las familias beneficiarias entre madres solteras, personas con capacidades especiales y adultos mayores en el año 2017.

La presente investigación se lo realiza en el sector rural de la Provincia de Cotopaxi, en las zonas más aledañas, donde se evidencia la pobreza cuyas familias sobreviven de los que la madre naturaleza les puede ayudar, con actividades agrícolas y sobre todo por el Bono de Desarrollo Humano que no todas las personas de esos sectores son los beneficiados, sino que viven en una lucha constante por acceder a este beneficio que el Gobierno ofrece a las familias de escasos recursos. Es así que la investigación se lo realiza a aquellas personas y verificar la incidencia que tienen estas transferencias monetarias que de alguna manera ayuda para conseguir el sustento diario y mantener un hogar.

$$
n=\frac{\left[Z^{2 *} N{ }^{*} p{ }^{*}\right]}{\left[B^{2 *}(N-1)+Z^{2 *} p{ }^{*} q\right]}
$$

Dónde:

$\mathrm{n}=$ muestra

$\mathrm{N}=$ población $(315)$

$\mathrm{p}=$ probabilidad de ocurrencia del suceso $(0.5)$

$\mathrm{q}=$ probabilidad de no ocurrencia del suceso (1 -

p) $Z=$ nivel de confianza $(95 \%=1.96)$

$\mathrm{B}=$ error de estimación ( $5 \%$ ) 
Por lo tanto, se tiene, un error de estimación del $5 \%$, una probabilidad de ocurrencia del suceso de 0.5 , una probabilidad de no ocurrencia del suceso (1p), un nivel de confianza de 1.96 teniendo así una muestra de 315 personas vulnerables para el estudio.

Se recolectó la información a través de la encuesta de la población beneficiaria del Bono de Desarrollo Humano en la provincia de Cotopaxi con preguntas acordes a los factores determinantes del problema. Para el análisis posterior de los resultados a través de un análisis estadístico los datos serán representados gráficamente utilizando el programa SPSS mediante gráficos de barras o pastel, el mismo que será mediante una representación gráfica el impacto del Bono de Desarrollo Humano en los beneficiados del sector rural de la Provincia de Cotopaxi.

\section{Resultados}

A continuación, se presenta la pregunta No. 8 del cuestionario aplicado a la muestra de las personas vulnerables en el sector rural de la Provincia de Cotopaxi ¿Cuál es el destino que usted le da al Bono de Desarrollo Humano? Con esta pregunta se quiere conocer el destino del Bono, dado que es importante conocer si ese dinero ocupa para las necesidades primordiales.

También se presenta la pregunta No. 9 del cuestionario aplicado a la muestra de las personas vulnerables en el sector rural de la Provincia de Cotopaxi ¿Ha mejorado su calidad de vida al beneficiarse del Bono de Desarrollo Humano? Con esta pregunta se quiere conocer si ha mejorado su estilo de vida con el Bono de Cotopaxi. Podemos decir que gran parte de los grupos vulnerables si ocupa el bono de desarrollo humano para sus necesidades básicas, es decir, el bono es fundamental en su economía.

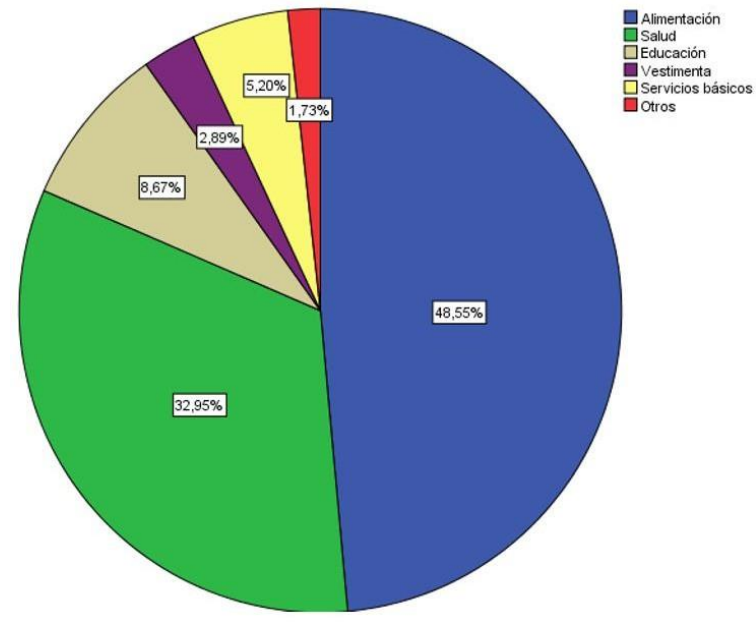

Gráfico 1. Destino del Bono

Fuente: Encuesta

Desarrollo, dado que es importante conocer las necesidades primordiales

Se logró identificar que el $68 \%$ de los beneficiarios encuestados aseguraron que el Bono de Desarrollo Humano, ha mejorado mucho su estilo de vida, seguido del $28 \%$ que mencionó que el bono ha mejorado poco su estilo de vida. Por otra parte, el $4 \%$ de los encuestados, mencionó que el bono no ha mejorado nada su estilo de vida. 


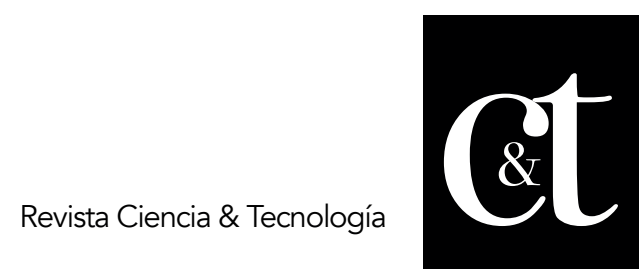

No. 24, 31 de octubre de 2019

ISSN impreso: 1390 - 6321

ISSN online: 2661 - 6734

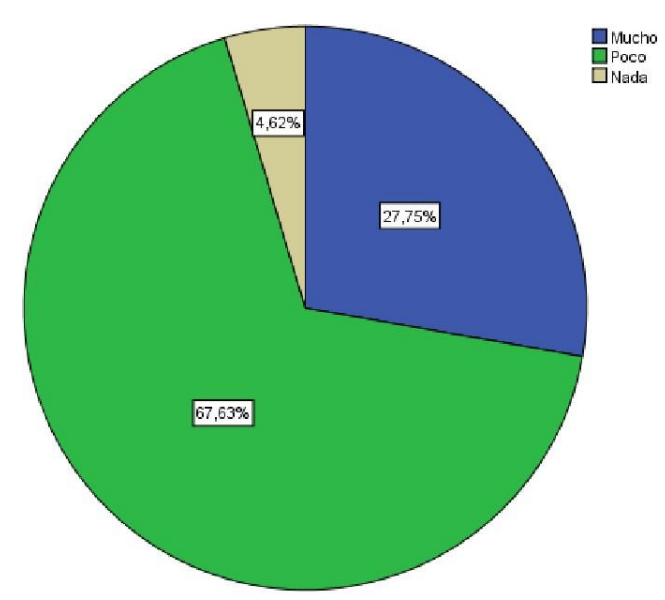

Gráfico 2. Estilo de vida

Fuente: Encuesta

Comprobación de hipótesis

$$
X^{2}=\frac{(o-e)^{2}}{e}
$$

La hipótesis al comprobarse en el presente estudio el bono de desarrollo humano incide en la calidad de vida de las personas vulnerables en el sector rural, donde se utilizó el estadígrafo chi cuadrado, que permite verificar si la hipótesis nula es cierta, es una prueba de bondad que se utilizó para el análisis de los datos categóricos de las variables en estudio. (Monge \& Pérez, 2015), manifiesta aplicar la siguiente fórmula:

$\mathrm{X} 2=$ Chi cuadrado $\mathrm{O}=$ Frecuencias observadas $\mathrm{E}=$ Frecuencias esperadas Se procede a calcular las frecuencias observadas, mostrada en la tabla No. 1 
Tabla 1. Contingencia de las frecuencias observadas

\begin{tabular}{|l|l|l|l|l|l|}
\multirow{2}{*}{$\begin{array}{l}\text { ¿Cuál es el } \\
\text { destino que }\end{array}$} & Alimentación & 36 & 42 & 6 & 84 \\
\cline { 2 - 7 } \begin{tabular}{l} 
usted le da \\
al Bono de \\
\cline { 2 - 7 }
\end{tabular} & Salud & 1 & 51 & 5 & 57 \\
\cline { 2 - 7 } $\begin{array}{l}\text { Desarrollo } \\
\text { Humano? }\end{array}$ & Eestimenta & 2 & 13 & 1 & 15 \\
\cline { 2 - 7 } & Servicios básicos & 4 & 1 & 2 & 5 \\
\hline Total & 1 & 2 & 0 & 3 \\
\hline
\end{tabular}

Fuente: Encuesta

Tabla 2. Contingencia de las frecuencias esperadas

\begin{tabular}{|c|c|c|c|c|c|}
\hline \multirow[t]{2}{*}{ Pregunta } & \multirow[t]{2}{*}{ Alternativas } & \multicolumn{3}{|c|}{$\begin{array}{l}\text { ¿Ha mejorado su calidad de } \\
\text { vida al beneficiarse del Bono de } \\
\text { Desarrollo Humano? }\end{array}$} & \multirow[t]{2}{*}{ Total } \\
\hline & & Mucho & Poco & Nada & \\
\hline \multirow{6}{*}{$\begin{array}{l}\text { ¿Cuál es el destino } \\
\text { que usted le da al } \\
\text { Bono de Desarrollo } \\
\text { Humano? }\end{array}$} & Alimentación & 21,8 & 54,4 & 7,8 & 84 \\
\hline & Salud & 14,8 & 36,9 & 5,3 & 57 \\
\hline & Educación & 3,9 & 9,7 & 1,4 & 15 \\
\hline & Vestimenta & 1,3 & 3,2 & 0,5 & 5 \\
\hline & Servicios básicos & 2,3 & 5,8 & 0,8 & 9 \\
\hline & Otros & 0,8 & 1,9 & 0,3 & 3 \\
\hline \multicolumn{2}{|l|}{ Total } & 45 & 112 & 16 & 173 \\
\hline
\end{tabular}

Fuente: Encuesta 


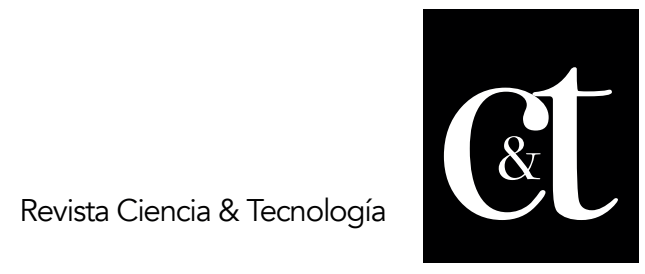

No. 24, 31 de octubre de 2019

ISSN impreso: 1390 - 6321

ISSN online: 2661 - 6734

Tabla 3. Cálculo del chi cuadrado

\begin{tabular}{|l|r|r|r|r|r|}
\hline Educación/Mucho & 1 & 3,9 & $-2,9$ & 8,4 & 2,2 \\
\hline Educación/Poco & 13 & 9,7 & 3,3 & 10,8 & 1,1 \\
\hline Educación/Nada & 1 & 1,4 & $-0,4$ & 0,1 & 0,1 \\
\hline Vestimenta/Mucho & 2 & 1,3 & 0,7 & 0,5 & 0,4 \\
\hline Vestimenta/Poco & 1 & 3,2 & $-2,2$ & 5,0 & 1,5 \\
\hline Vestimenta/Nada & 2 & 0,5 & 1,5 & 2,4 & 5,1 \\
\hline Servicios básicos/Mucho & 4 & 2,3 & 1,7 & 2,8 & 1,2 \\
\hline Servicios básicos/Poco & 3 & 5,8 & $-2,8$ & 8,0 & 1,4 \\
\hline Servicios básicos/Nada & 2 & 0,8 & 1,2 & 1,4 & 1,6 \\
\hline Otros/Mucho & 1 & 0,8 & 0,2 & 0,0 & 0,1 \\
\hline Otros/Poco & 2 & 1,9 & 0,1 & 0,0 & 0,0 \\
\hline Otros/Nada & 0 & 0,3 & $-0,3$ & 0,1 & 0,3 \\
\hline Total & & & & & 45,6 \\
\hline
\end{tabular}

Fuente: Encuesta

Luego del cálculo de las frecuencias observadas y las frecuencias esperadas por medio de las tablas de contingencia se procedió al cálculo del chi cuadrado, en este caso el chi cuadrado fue de 45.6. Por ende, se calcula los grados de libertad dependiendo las filas y columnas trabajadas en este caso se trabajó con 6 filas y 3 columnas, dando un resultado de 10. Dado que los grados de libertad son 10 con un $a=0.05$ tenemos $x 2 t=18,3070$. Entonces se debe comparar la condición de aceptación del chi cuadrado calculado representada por $\times 2 \mathrm{c}$ y el chi cuadrado tabulado representada por x2t. Según Monge Ivars \& Pérez, (2015) manifiesta que si se cumple la condición chi cuadrado calculado es mayor a chi cuadrado tabulado se rechaza la hipótesis nula y se acepta la hipótesis alternativa en este caso $\times 2 c=45.6$ que es mayor a $\times 2 t$ que es 18,3070 .

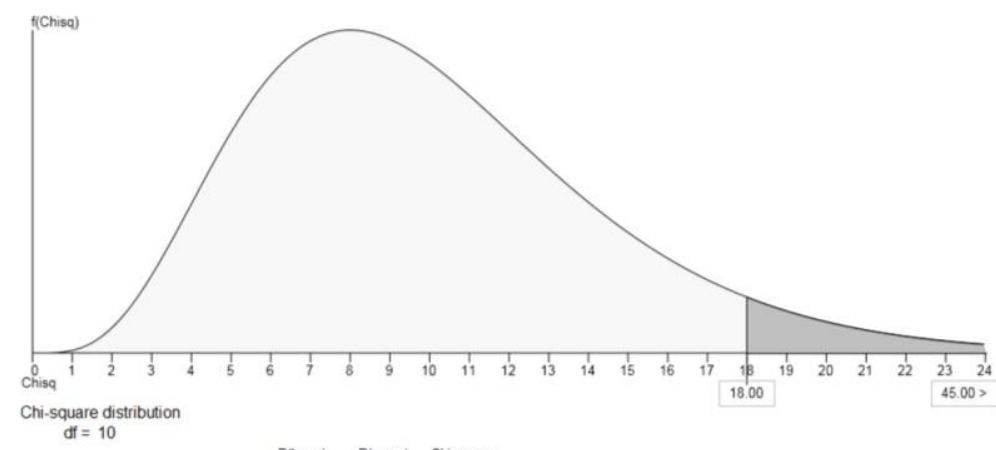

df $=10$

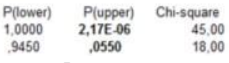

Gráfico 3. Campana de Gauss

Fuente: Autores 


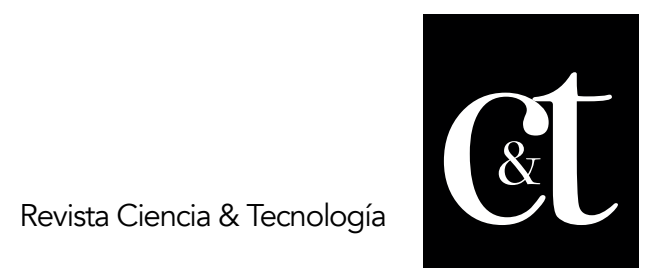

No. 24, 31 de octubre de 2019

ISSN impreso: 1390 - 6321

ISSN online: 2661 - 6734

La decisión final con el Chi cuadrado calculado 45,6 es mayor que el Chi cuadrado tabulado 18,3070 , a 10 grados de libertad y una tolerancia máxima de error del 0.05 a un nivel de confianza del $95 \%$, por consiguiente, se rechaza la hipótesis nula y se acepta la hipótesis alterna, es decir que "El bono de desarrollo humano sí incide en la calidad de vida de las personas vulnerables en el sector rural".

\section{Conclusiones}

Con el objetivo de identificar la función que cumple el Bono de Desarrollo Humano en la economía de los beneficiarios, se concluye que el destino que le da el beneficiario a este monto de dinero que emite el estado ecuatoriano, es la alimentación (49\%), seguido por la salud (33\%) y la educación ( $9 \%$ ). Actividades que forman parte de las necesidades básicas de la población.

En relación, a la calidad de vida del beneficiario, este beneficio social poco o nada ha mejorado su situación dado que consideran que el monto mensual que perciben es relativamente bajo, valor que aporta en algo a la economía de la persona pero que no alcanza para cubrir todas las necesidades básicas y más aún si esta posee cargas familiares, personas enfermas o personas con discapacidad.

Finalmente, se determinó que existe una correlación directa entre la variable bono de desarrollo humano y calidad de vida dado que estadísticamente a través del método Ji cuadrado se cumplió la relación $x c 2>x t 2$ tomando en cuenta los grados de libertad, se pudo aceptar la hipótesis alternativa planteada en el presente estudio, por lo tanto, el bono de desarrollo humano si incide en el estilo de vida de las personas vulnerables en el sector rural.

\section{Referencias bibliográficas}

Arias Cortés, B. P. (2013). El concepto de calidad de vida en las teorías del desarrollo. Criterio Jurídico Garantista. Vol. 5(8); pp. 136-149.

Cantú Martínez, P. C. (2015). Calidad de vida y sustentabilidad: una nueva ciudadanía. Ambiente y Desarrollo. Vol. 19(37); pp.9-22

Cardona, D., \& Agudelo, H. B. (2005). Construcción cultural del concepto calidad de vida. Revista Facultad Nacional de Salud Pública. Vol. 23(1); pp. 79-90.

Celemin, J. P., Mikkelsen, C. A., \& Velázquez, G. Á. (2015). La calidad de vida desde una perspectiva geográfica: integración de indicadores objetivos y subjetivos. Revista Universitaria de Geografía. Vol. 24; pp. 63-84.

Gómez Álvarez, R., Morales Sánchez, R., \& Rodriguez Morilla, C. (2017). La Economía del Bien Común en el ámbito local. Revista de Economía Pública, Social y Cooperativa. Vol. 90; pp. 189-222.

Martínez Dobronsky, J., \& Rosero Moncayo, J. (2015). Impacto del Bono de Desarrollo Humano en el Trabajo Infantil. MIES, Quito: Ecuador.

Monge Ivars, J. F., \& Pérez, J. (2015). Estadística no paramétrica, prueba chi cuadrado. Proyecto e-Math. Universidad Oberta de Catalunya. España. 


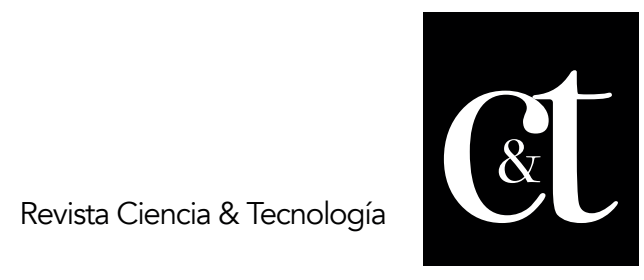

No. 24, 31 de octubre de 2019

ISSN impreso: 1390 - 6321

ISSN online: 2661 - 6734

Rodriguez, V., Rojo, F., \& Fernández, G. (2011). Recursos económicos y calidad de vida en la población mayor. Revista Internacional de Sociología (RIS). Vol. 69(1); pp. 195-227.

Roura Camacho, C. J. (2016). Análisis socioeconómico de los beneficiarios del Bono de Desarrollo Humano (BDH) a diciembre 2015 y elementos de política complementarios. Tesis de grado. PUCE: Quito, Ecuador.

Troya Rodríguez, J. V. (2014). El desarrollo sostenible enfoques desde la economía. Apuntes del CENES. Vol. 35(62); pp. 15-52.

Vázquez Maguirre, M. (2014). La empresa social como detonadora de calidad de vida y desarrollo sustentable en comunidades rurales. Pensamiento y gestión, № 37, 30 ; pp. 256-285. 